\title{
PRECLINICAL EFFECTIVENESS OF AN EXPERIMENTAL TRICALCIUM SILICATE CEMENT ON PULPAL REPAIR
}

\author{
Xin $\mathrm{Li}^{1 *}$, Mariano Simón Pedano ${ }^{1 *}$, Shuchen $\mathrm{Li}^{2}$, Zheyi Sun ${ }^{2}$, Charlotte Jeanneau ${ }^{3}$, Imad \\ About $^{3}$, Esther Hauben ${ }^{4}$, Zhi Chen ${ }^{2}$, Kirsten Van Landuyt ${ }^{1}$, Bart Van Meerbeek ${ }^{1}$
}

${ }^{1}$ KU Leuven (University of Leuven), Department of Oral Health Sciences, BIOMAT \& UZ Leuven (University Hospitals Leuven), Dentistry, Leuven, Belgium;

${ }^{2}$ Wuhan University, School and Hospital of Stomatology, The State Key Laboratory Breeding Base of Basic Science of Stomatology (Hubei-MOST) \& Key Laboratory of Oral Biomedicine of Ministry of Education, Wuhan, PR China;

${ }^{3}$ Aix Marseille Univ, CNRS, ISM, Inst Movement Sci, Marseille, France;

${ }^{4}$ Laboratory for Pathology, UZ Leuven \& Department of Imaging and Pathology, translational cell and tissue research, $K U$ Leuven.

*Equal first-author contribution.

Corresponding author: Prof. Dr. B. Van Meerbeek, KU Leuven (University of Leuven), Department of Oral Health Sciences, BIOMAT, Kapucijnenvoer 7, blok a - box 7001, BE-3000 Leuven, Belgium. TEL: +32-16-337587, FAX: +32-16-332752, bart.vanmeerbeek@kuleuven.be 


\section{ABSTRACT}

Objectives: To investigate the pulpal repair potential of an experimental zirconium-oxide containing tricalcium-silicate cement, referred to as 'TCS 50 '.

Materials and methods: The effect of TCS 50 on viability, proliferation, migration, and odontoblastic differentiation of human dental pulp cells (HDPCs) was assessed using XTT assay, in-vitro wound healing assay and RT-PCR, respectively. Additionally, the pulp-capping potential was evaluated using a vital human tooth model. Statistical analysis was performed using non-parametric Kruskal-Wallis test and post-hoc test (Mann-Whitney $U$ test). The tests were performed at a significance level of $\alpha=0.05$. Results: The effect of TCS 50 towards HDPCs was dose dependent. Undiluted TCS 50 extract showed no immediate adverse impact on cell viability $(p>0.05)$; however, it significantly inhibited proliferation and migration of HDPCs $(p<0.05)$. A $25 \%$ diluted TCS 50 extract showed no significant effect on cell viability, proliferation or migration ( $p>0.05$ ), and it significantly enhanced odontoblastic differentiation of HDPCs $(p<0.05)$. In pulps capped with TCS 50 for both 2 and 4 weeks, H\&E staining revealed a normal morphology of pulp tissue; mineralized foci with cellular components entrapped in the matrix were formed underneath the exposure site. Collagen I expression was weak within the matrix of mineralized foci, while the expression of nestin was positive for entrapped cellular components within the mineralized foci, indicating that the formed mineralized foci corresponded to an initial form of reparative dentin formation.

Conclusion: TCS 50 is capable of generating an early pulp-healing reaction and therefore could serve as a promising pulp-capping agent.

Keywords: tricalcium-silicate cement, pulp, odontoblast, tooth model 


\section{INTRODUCTION}

Hydraulic calcium-silicate cements ( $\mathrm{hCSCs}$ ), for instance the most known and most intensively investigated hCSC referred to as 'Mineral Trioxide Aggregate' ('MTA'), have widely been used as pulpcapping agents. Compared with the gold-standard pulp-capping material calcium hydroxide, hCSCs induce less pulp inflammatory reaction and more predictably form reparative dentin $[1,2]$. However, the shortcomings of hCSCs, for instance their difficult handling property $[3,4]$, long setting time [5], heavy metal element release [6], as well as tooth-discoloration potential [7], remain a concern.

To overcome the abovementioned drawbacks, an experimental zirconium-oxide $\left(\mathrm{ZrO}_{2}\right)$ containing tricalcium-silicate (TCS) based cement, referred to as TCS 50, has been developed. TCS 50 powder consists of 50 wt $\%$ TCS and 50 wt $\% \mathrm{ZrO}_{2}$; to accelerate the setting process, $1 \mathrm{M} \mathrm{CaCl}_{2}$ was applied as liquid. Our previous study showed that TCS 50 demonstrated a mini-fracture toughness comparable to that of a commercial TCS-based cement Biodentine (Septodont, Saint Maur des Fosses, France), which consists of TCS and dicalcium silicate as the main active components, and $\mathrm{ZrO}_{2}$ as a radiopacifier. The release of Ca from TCS 50 was initially slowed down but prolonged up to 1 month; additionally, TCS 50 was cytocompatible towards human dental pulp fibroblasts [8]. Moreover, our on-going study showed that freshly mixed TCS 50 revealed an alkaline $\mathrm{pH}$ of 12.0 , this initially at $1 \mathrm{~h}$ after mixing; this $\mathrm{pH}$ remained stable for $24 \mathrm{hrs}$. The $28-\mathrm{d}$ cumulative release of Ca and Si from freshly mixed TCS 50 reached approximately 3000 and 2000 ppm, respectively. Accordingly, the formulation of the experimental hCSC TCS 50 was thought to be a promising pulp-capping agent.

The pulp-repair process starts with the recruitment and migration of stem/progenitor cells to the injury/exposure sites, followed by the proliferation and stimulation of stem/progenitor cells to differentiate into odontoblastic-like cells; the odontoblastic-like cells then secrete an extracellular matrix which serves as a scaffold for the formation of reparative dentin to seal the exposure [9]. The ideal pulp-capping material should, therefore, facilitate the proliferation, migration and odontoblastic differentiation of stem/progenitor cells [10]. In continuation of previous research, the effect of TCS 50 on the viability, proliferation, migration and odontoblastic differentiation of human dental pulp cells (HDPCs) was assessed. Different from most previous studies, which commonly tested cements in their set state $[11,12]$, freshly mixed cements were investigated in this study to better mimic the clinical condition, this following a protocol also employed in our previous study [13].

Apart from in-vitro cellular research, in-vivo laboratory animal models, for instance rats $[14,15]$, dogs [16,17], ferrets [18], monkeys [19], and miniature pigs [20,21], have widely been used to evaluate the preclinical effectiveness of pulp-capping agents. Nevertheless, the pulp-capping success rate in animal teeth appeared not always very alike that found clinically in human teeth; this should be attributed to differences in the anatomical and physiological characteristics of the dentitions $[22,23]$. In addition, the related ethical issues as well as the high cost of animal studies should be considered. 
Recently, an innovative vital human tooth model has been developed to assess pulp-capping effectiveness [24,25]. Briefly, the pulp tissue of a freshly extracted immature human third molar was mechanically exposed, upon which the exposure was capped with a pulp-capping agent. The tooth was cultured in vitro up to 2 or 4 weeks to observe the pulpal response in contact with the pulp-capping agent. In pulps capped with the commercial cements ProRoot MTA (Dentsply Sirona, Konstanz, Germany) and Biodentine (Septodont), the formation of mineralized foci with osteodentin morphology was observed early at 2 weeks after pulp capping [25]. This preclinical laboratory model provides insights into the early response of pulp tissue exposed to pulp-capping agents.

The objective was therefore (1) to characterize the effect of TCS 50 on the viability, proliferation, migration, and odontoblastic differentiation of HDPCs, and (2) to evaluate the pulpal response in pulps capped with TCS 50 using a vital human tooth model. The market-representative hCSCs ProRoot MTA (Dentsply Sirona) and TheraCal LC (Bisco, Schaumburg, IL, USA) were tested as resin-free and resinbased references, respectively. The null hypotheses tested were that (1) TCS 50 showed no effect on the viability, proliferation, migration, and odontoblastic differentiation of HDPCS, (2) TCS 50 did not display any pulpal reaction in exposed pulps after 2 or 4 weeks culturing, and (3) the effects of TCS 50 onto HDPCs and exposed human pulps were not favorable as compared to those of the reference resinfree cement ProRoot MTA (Dentsply Sirona) and resin-based cement TheraCal LC (Bisco).

\section{MATERIALS \& METHODS}

\subsection{In-vitro cellular assessment}

\subsubsection{HDPCs isolation and characterization}

Healthy human third molars (from patients at the age of 15-25 years old) extracted for orthodontic reasons, were collected as approved by the Commission for Medical Ethics of KU Leuven (S54254). HDPCs were isolated from the pulp tissue following the out-growth method. The isolated cells were subsequently characterized with immunofluorescence staining, flow cytometry, colony-forming assay and multiple lineage differentiation assay. The protocols employed were described in detail in our previous study [13]. Cells at passages 3-6 were used for this study.

\subsubsection{Preparation of cement eluates}

Three cements, namely TCS 50, ProRoot MTA (Dentsply Sirona) and TheraCal LC (Bisco) were tested. The cement eluates were prepared using sterile instruments under a laminar flow hood. TCS 50 was produced in house, the cement powder consists of $50 \mathrm{wt} \%$ TCS (diameter: $\pm 10 \mu \mathrm{m}$; Mineral Research Processing, Meyzieu, France) and 50 wt $\% \mathrm{ZrO}_{2}$ (diameter: \pm 200 nm; Tosoh, Tokyo, Japan). Cement powder was mixed with $1 \mathrm{M} \mathrm{CaCl}_{2}$ (Sigma-Aldrich, St. Louis, MO, USA) at a weight ratio of 3:1 for 30 sec (RotoMix Capsule Mixer, 3M Oral Care, Seefeld, Germany). The reference cements were prepared 
according to the manufacturers' instructions. Next, $0.3-\mathrm{cm}^{3}$ freshly mixed cement was applied directly onto the bottom of a well of a 12-well plate (diameter: $22.1 \mathrm{~mm}$ ); the cement was compacted with a sterile cotton swab until it homogenously covered the bottom surface of the well. The resin-based cement TheraCal LC (Bisco) was light-cured with a LED curing light (Bluephase 20i, Ivoclar Vivadent, Schaan, Liechtenstein) at an output of $1200 \mathrm{~mW} / \mathrm{cm}^{2}$ for $20 \mathrm{sec}$, the intensity of the light was measured using a Marc Resin Calibrator (BlueLight Analytics, Halifax, Canada). 3 mL Dulbecco's Modified Eagle Medium (DMEM; Gibco, Carlsbad, CA, USA), supplemented with or without $10 \%$ fetal bovine serum (FBS; Gibco), was added to each well, and the cement immersed in the medium was incubated at $37^{\circ} \mathrm{C}$, $5 \% \mathrm{CO}_{2}$ and $95 \%$ humidity. The medium was collected for the first time after 24 hrs of incubation, having been regarded as the 1-day cement eluate. Subsequently, $3 \mathrm{~mL}$ fresh medium was added to the same well. The medium was refreshed every 3 days to mimic the in-vivo dynamic refreshing condition. Therefore, 4-, 7-, 10- and 14-day cement eluates were collected, respectively. Three dilutions (50\%, $25 \%$ and $10 \%$ ) of the corresponding cement eluates were prepared at each time interval.

\subsubsection{Cell-viability assay}

To assess the cell viability, an XTT assay was used, this based on the cleavage of the tetrazolium salt XTT (Sigma-Aldrich) by mitochondrial enzymes in viable cells to form the soluble formazan. The amount of formazan produced directly correlates to the number of metabolically active cells. HDPCs were seeded at a concentration of $1.5 \times 10^{4}$ cells/well in $100 \mu \mathrm{L}$ culture medium in 96-well plates, and then incubated at $37^{\circ} \mathrm{C}$ in $5 \% \mathrm{CO}_{2}$ for $24 \mathrm{hrs}$. Culture medium was subsequently replaced with $50 \mu \mathrm{L}$ undiluted/diluted 1-day cement eluates. After $20 \mathrm{hrs}$ incubation and $4 \mathrm{hrs}$ before spectrophotometric analysis, $50 \mu \mathrm{L}$ XTT labeling agent and electron-coupling reagent were added to each well. The amount of formazan produced was quantified at a wavelength of $450 \mathrm{~nm}$ using a microplate reader (Multiskan Ascent 96/384, Thermo Fisher Scientific, Waltham, MA, USA). The formazan productions in cells, which were cultured in culture medium or $1 \%$ Triton X-100, were employed as negative and positive controls, respectively. The corresponding absorbance values were adjusted to 100 and $0 \%$, upon which the relative formazan production was calculated. ZnO-eugenol cement (Alganol, Kemdent, Swindon, UK) was tested as positive control. Cement eluates at every dilution (undiluted, 50\%, 25\% and 10\%) were assessed 3 times per test, and the test was repeated in triplicate with cells from 3 different donors.

\subsubsection{Cell-proliferation assay}

To assess the proliferation trend of HDPCs having been exposed to different cement eluates, XTT assays were performed at three different time intervals, namely at day 1,4 and 7. HDPCs were seeded at a low concentration of 1,000 cells/well, and the medium in contact with cells was refreshed every 3 days with the corresponding undiluted/diluted cement eluates. As for the cell-viability assay, the formazan 
productions in HDPCs that were cultured in culture medium or 1\% Triton X-100 (Sigma-Aldrich), were used as negative and positive controls, respectively. The absorbance values of the positive and negative controls at day 7 were adjusted to $0 \%$ and 100\%, upon which the relative formazan productions at day 1, 4 and 7 were calculated. Each cement extract at every dilution (undiluted, 50\%, $25 \%$ and $10 \%$ ) was tested 3 times per test, and the test was repeated in triplicate with cells from 3 different donors.

\subsubsection{Cell-migration assay: in-vitro wound-healing assay}

HDPCs were seeded into a 12 -well plate at a density of $2 \times 10^{5}$ cells/well. Cells were starved in serumfree DMEM (Gibco) for 24 hrs after reaching 90\% confluence. In each well, a scratch was made using the tip of a sterile $100 \mu \mathrm{L}$ pipette. The cells were then washed with phosphate buffered saline (PBS, Gibco), upon which the cells were incubated with diluted/undiluted 1-day cement eluates in the absence of FBS (Gibco) for $24 \mathrm{hrs}$. Microphotographs of 3 randomly selected fields of the scratch were taken at 0 and $24 \mathrm{hrs}$ post-wound induction under a light microscope (Primo Vert, Carl Zeiss, Jena, Germany) at a 100x magnification. The number of cells which were migrated to the scratched area after $24 \mathrm{hrs}$ was counted independently by two researchers. HDPCs that were exposed to serum-free DMEM (Gibco), were selected as blank control. This experiment was repeated 6 times with cells from 3 different donors.

\subsubsection{Odontoblastic differentiation assay: real-time polymerase chain reaction (RT-PCR)}

To evaluate the odontoblastic differentiation of HDPCs, three representative genes, namely alkaline phosphatase (ALP), dentin sialophosphoprotein (DSPP), and osteocalcin (OCN) were selected. The cells were seeded at a density of $2 \times 10^{5}$ cells/well in 6 -well plates, they were cultured with DMEM (Gibco) containing 10\% FBS (Gibco), till reaching $80-90 \%$ confluence. The culture medium was then replaced by 1 -day $25 \%$ diluted cement eluates. The cells were cultured for 4,10 or 14 days with the cement eluates being refreshed every 3 days. After culturing, the total RNA of the cells, being exposed to cement eluates, was collected using a E.Z.N.A Total RNA Kit I (Omega BIO-TEK, Norcross, GA, USA). The purity and concentration of the extracted RNA was assessed using a spectrophotometer (SimpliNano, Biochrom, Cambridge, UK). First-strand cDNA was generated with a RevertAid First Strand cDNA Synthesis Kit (Thermo Fisher Scientific) using a 9700 PCR system (Applied Biosystems, Foster City, CA, USA). RT-PCR was performed in a CFX 384 Touch Real-time PCR Detection System (Bio-Rad, Hercules, CA, USA) with PowerUp SYBR Green Master Mix (Thermo Fisher Scientific). For each $20 \mu \mathrm{L}$ volume reaction, $1 \mu \mathrm{L}$ cDNA was used. The amplification process started with an initial step at $95^{\circ} \mathrm{C}$ for $10 \mathrm{~min}$, followed by 40 cycles of $\mathrm{PCR}$, comprising denaturation for $15 \mathrm{sec}$ at $95^{\circ} \mathrm{C}$ and combined annealing/extension for $1 \mathrm{~min}$ at $60^{\circ} \mathrm{C}$, and was finished at $95^{\circ} \mathrm{C}, 60^{\circ} \mathrm{C}$ and $95^{\circ} \mathrm{C}$ for $15 \mathrm{sec}$ each. Cells 
which were exposed to odontogenic differentiation medium, consisting of DMEM supplemented with $50 \mu \mathrm{g} / \mathrm{mL}$ ascorbic acid (Sigma-Aldrich), $10 \mathrm{mM}$ sodium $\beta$-glycerophosphate (Sigma-Aldrich), $10 \mathrm{nM}$ dexamethasone (Sigma-Aldrich) and 10\% FBS (Gibco), were selected as positive control. The gene expressions of ALP, DSPP, and OCN were defined from the threshold cycle (CT), and the expression levels were calculated using the $2^{-} \Delta^{\mathrm{CT}}$ method after the normalization with reference to the expression of the housekeeping gene glyceraldehyde 3-phosphate dehydrogenase (GAPDH). The relative expressions of ALP, DSPP, and OC from HDPCs, having been exposed to cement eluates, were calculated as a percentage of the corresponding gene expression in the positive control group. The sense and antisense primers (Integrated DNA Technologies, Leuven, Belgium) used for RT-PCR are listed in Table 1. The experiment was performed three times and the assay was repeated in triplicate with HDPCs from 3 different donors.

\subsection{Vital human tooth model}

\subsubsection{Pulp-capping procedures}

Twenty-four healthy human immature third molars (from patients at the age of 15-18 years old), with one- to two-third root formations as well as open apices (Fig. 1a) and extracted for orthodontic reasons, were collected. Immediately after extraction, the teeth were immersed in DMEM (Gibco) supplemented with 10\% FBS (Gibco), $200 \mathrm{U} / \mathrm{mL}$ penicillin, and $200 \mu \mathrm{g} / \mathrm{mL}$ streptomycin (Invitrogen, Carlsbad, CA, USA), and transported to the laboratory. The following procedures were performed under a laminar flow cabinet and the operators wore gloves, masks and magnifying glasses. The periodontal ligament of each tooth was eliminated using a sterile \#15 blade (Swann Morton, Sheffield, UK). The tooth was thoroughly rinsed using PBS (Gibco) with $200 \mathrm{U} / \mathrm{mL}$ penicillin and $200 \mu \mathrm{g} / \mathrm{mL}$ streptomycin (Invitrogen). After rinsing, the tooth was handled with sterile gauze (Yibon Medical, Kuurne, Belgium) soaked in DMEM medium (Gibco) containing 10\% FBS (Gibco), $200 \mathrm{U} / \mathrm{mL}$ penicillin and $200 \mu \mathrm{g} / \mathrm{mL}$ streptomycin (Invitrogen) to avoid desiccation. A standard class-I cavity was prepared at the occlusal surface of each tooth using a sterile diamond bur (diameter: $1.1 \mathrm{~mm}$; A 0164300001 00, Dentsply Sirona) at an ultra-high speed with sterile saline (Fresenius Kabi, Bad Homburg, Germany) as a coolant. The pulp exposure was prepared with a sterile carbide bur (diameter: $1.0 \mathrm{~mm}$; H1SE.205.010, Komet, Lemgo, Germany) (Fig. 1b). The cavity was rinsed thoroughly with saline (Fresenius Kabi), and hemorrhage, if occurred, was controlled using sterile cotton pellets. The tooth crown was also gently dried with sterile cotton pellets, upon which the pulp exposure (diameter: 1.0 $\mathrm{mm}$ ) was sealed by pulp-capping agents at a thickness of $2 \mathrm{~mm}$ using a calcium-hydroxide applier (Ash Lustra Excavator, Dentsply Sirona) (Fig. 1c). A layer of resin-based glass-ionomer cement (Fuji II LC Capsule, GC, Tokyo, Japan) was applied on top of the pulp-capping agent. The cavity was then restored with a universal adhesive (G-Premio Bond, GC) and a restorative composite (G-aenial Posterior, GC). 
The crown of the tooth was fixed to a sterile stainless standard square orthodontic wire $(0.41 \times 0.41$ mm, REF 251-616, 3M Unitek, Monrovia, CA, USA). The tooth was cultured in a well of a 24-well plate (Costar, Cambridge, MA, USA) with the wire being suspended on the upper border of the well. To facilitate diffusion of the culture medium through the apical part, the apical part of the tooth was kept away from the bottom of the well. The root of the tooth was immersed in $1.5 \mathrm{~mL}$ DMEM (Gibco) containing 10\% FBS (Gibco), $200 \mathrm{U} / \mathrm{mL}$ penicillin, and $200 \mu \mathrm{g} / \mathrm{mL}$ streptomycin (Invitrogen) (Fig. 1d). Exposed pulps without capping were cultured under the same condition to serve as blank control. The teeth were cultured for 2 or 4 weeks ( $n=3$ per group and per material), with the culture medium daily refreshed. The used culture medium was re-collected and further checked with brain heart infusion (BHI) on a regular basis (every 3 days) to detect any sign of contamination. After culturing, each tooth was fixed in $30 \mathrm{~mL} 4 \%$ paraformaldehyde solution (VWR International, Leuven, Belgium) at $4^{\circ} \mathrm{C}$ for 2 weeks.

\subsubsection{Histological evaluation}

After fixation, the teeth were decalcified at room temperature $\left(22^{\circ} \mathrm{C}\right)$ in a decalcifier (Surgipath Decalcifier II, Leica Biosystems, Wetzlar, Germany). The decalcification solution was refreshed every 3 days, the endpoint was determined using a dental radiography (MINRAY, Soredex, Tuusula, Finland). After decalcification, the composite was manually removed, upon which setting of the cements was confirmed before the teeth were washed under running water for $24 \mathrm{hrs}$, and dehydrated in ethanol ( $70 \%$ for $12 \mathrm{hrs}, 80 \%, 95 \%$ and $100 \%$ for $2 \mathrm{hrs}$ each), this followed by xylene clearance. To facilitate the penetration of paraffin, the teeth were firstly immersed in liquid paraffin (Klinipath, Duiven, The Netherlands) for $24 \mathrm{hrs}$ and then embedded in paraffin blocks.

The teeth were sagittally sectioned at a thickness of 5-7 $\mu \mathrm{m}$, beginning at the level at which the pulp-capping agent firstly interacted with pulp tissue till the interaction was no longer available, this using a microtome (Microm HM 360 Microtome, Hyland Scientific, Stanwood, WA, USA). Four sections were randomly selected from each 12 sequential sections. One section was stained with Gill's III hematoxylin and $1 \%$ aqueous solution eosin (Leica Microsystems, Diegem, Belgium) (H\&E); one section was stained with Gram-Twort for bacterial recognition, and the remaining two sections were processed for immunohistochemical characterization of collagen I (anti-collagen I antibody COL-1, ab6308, Abcam, Cambridge, UK) and nestin (anti-nestin antibody 10C2, ab22035, Abcam), respectively. For each tooth, approximately 32 sections were stained, with 8 sections per staining. The sections were evaluated under a light microscopy (Axio Imager M2, Carl Zeiss Microscopy, Jena, Germany). 


\subsection{Statistical analysis}

Statistical analysis was performed using non-parametric Kruskal-Wallis test and post-hoc analysis (Mann-Whitney $U$ test). The tests were performed at a significance level of $\alpha=0.05$.

\section{RESULTS}

\subsection{In-vitro cellular assessment}

\subsubsection{Cell-viability assay}

The relative formazan formation by HDPCs after exposure to undiluted $(100 \%)$ and diluted $(50 \%, 25 \%$ and $10 \%$ ) cement eluates are presented in Fig. 2. For undiluted cement eluates, cells cultured with TCS 50 and ProRoot MTA eluates showed relatively high formazan production of $99.7 \pm 11.5 \%$ and $102.1 \pm 3.1 \%$, respectively, indicating that the cements were cytocompatible. TheraCal LC and $\mathrm{ZnO}$ eugenol cement undiluted eluates, however, largely decreased the relative formazan formation, and this difference is significant $(p<0.05)$. The diluted TCS 50 eluates $(50 \%, 25 \%$ and $10 \%)$ showed comparable cytocompatibility as that of the undiluted TCS 50 eluate $(p>0.05)$. The cytocompatibility of $50 \%$ and $25 \%$ diluted eluates was in decreasing order as follows: TCS 50, ProRoot MTA > TheraCal LC > ZnO-eugenol cement (Fig. 2). For 10\% ZnO-eugenol cement dilutions, the cytocompatibility increased to a level comparable with that of TheraCal LC $(p>0.05)$, yet being significantly lower than that of TCS 50 and ProRoot MTA ( $p<0.05)$ (Fig. 2).

\subsubsection{Cell-proliferation assay}

The relative formazan formation by HDPCs having been exposed to undiluted $(100 \%)$ and diluted $(50 \%$, $25 \%$ and $10 \%$ ) cement eluates for 1, 4 and 7 days are summarized in Fig. 3. The relative formazan formation of cells exposed to culture medium (negative control) increased progressively from $18.7 \pm 6.0 \%$ at day 1 to $82.3 \pm 9.6 \%$ at day 4 till $99.5 \pm 4.1 \%$ at day 7 ; the differences were statistically significant $(p<0.05)$. The relative formazan formation by cells exposed to TCS 50 undiluted eluate also significantly increased over time $(p<0.05)$, however, the value was significantly lower than that of the culture medium at day 4 and $7(p<0.05)$. For all TCS 50 diluted eluates $(50 \%, 25 \%$ and $10 \%)$, the relative formazan formation was significantly lower than that of the control at day $4(p<0.05)$; however, the values all increased to a comparable level as to that of the control at day $7(p>0.05)$. The proliferation trend of cells exposed to ProRoot MTA undiluted 50\% diluted eluates resembled closely that of TCS 50; no significant difference was found at any time interval ( $p>0.05$ ). For $25 \%$ and $10 \%$ diluted eluates, cells exposed to ProRoot MTA showed significantly higher levels of relative formazan formation than that of TCS 50 at day $4(p<0.05)$, while at day 7 , no significant difference could be detected $(p>0.05)$. Regarding TheraCal LC undiluted eluates, the relative formazan formation was significantly lower than that of TCS $50(p<0.05)$, and the value significantly decreased over time $(p<0.05)$, indicating cytotoxicity 
of the TheraCal LC cement eluates. The relative formazan formation for TheraCal LC diluted eluates (50\%, $25 \%$ and $10 \%)$ were all significantly lower than that of TCS $50(p<0.05)$, except for TheraCal LC $25 \%$ and $10 \%$ dilution at day 1 ( $p>0.05)$. ZnO-eugenol undiluted and diluted cement eluates also showed significantly lower levels of relative formazan formation as compared to TCS $50(p<0.05)$; the only exception was ZnO-eugenol 10\% diluted cement eluate at day 1 ( $p>0.05)$.

\subsubsection{Cell-migration assay}

The number of migrated cells exposed to 1-day undiluted (100\%) and diluted (50\%, $25 \%$ and $10 \%)$ cement eluates are shown in Fig. 4. For undiluted cement eluates, the number of migrated cells was significantly lower than that of the culture medium control $(p<0.05)$, regardless of the cement evaluated. For diluted cement eluates $(50 \%, 25 \%$ and $10 \%)$, the number of migrated cells for TCS 50 was comparable to that of the control $(p>0.05)$, and no significant difference was detected for different eluate concentrations $(p>0.05)$. ProRoot MTA diluted eluates all significantly increased the number of migrated HDPCs compared to that of the control $(p<0.05)$. TheraCal LC dilutions, however, all significantly decreased the number of migrated cells $(p<0.05)$. Representative photomicrographs of the scratched area in cells, which were exposed to $25 \%$ diluted cement eluates, are shown in Fig. 5 . For cells exposed to TCS 50 (Fig. 5a,b), ProRoot MTA (Fig. 5c,d) and the culture-medium control (Fig. $5 \mathrm{~g}, \mathrm{~h})$, a typical fibroblast-like morphology with the presence of multiple elongated processes was observed. The number of migrated cells was the highest for ProRoot MTA (Fig. 5d), this followed by the culture-medium control (Fig. 5h) and TCS 50 (Fig. 5b). For TheraCal LC, HDPCs appeared in a ballshape morphology (Fig. 5e,f), while no cell migration was observed (Fig. 5f).

\subsubsection{Odontoblastic differentiation assay}

The cell-viability and cell-proliferation assays showed that the TheraCal LC diluted eluates $(50 \%, 25 \%$ and $10 \%$ ) were all cytotoxic towards HDPCs. The cell-migration assay revealed that the $50 \%$ and $25 \%$ cement dilutions were most appropriate to detect odontoblastic differentiation potential of the cements. Since in literature the information regarding the resin-based cement TheraCal LC (Bisco) is insufficient, a lower and less toxic concentration (25\% dilution) was selected, this to provide more insights into the performance of TheraCal LC (Bisco).

The relative expressions of ALP, DSPP, and OCN from HDPCs, cultured with $25 \%$ cement dilution for 4, 10 and 14 days are presented in Fig. 6. For cells exposed to TCS $5025 \%$ diluted cement eluate, the gene expressions of ALP, DSPP, and OCN at day 4 were all significantly lower than that of the cells cultured with odontogenic differentiation medium control $(p<0.05)$. However, at day 10 , all gene expressions reached higher levels than of the control, and the differences were significant $(p<0.05)$. At day 14, ALP expression was significantly lower than of the control $(p<0.05)$, while DSPP and OCN 
expressions were still significantly higher $(p<0.05)$. Similar as TCS 50 , ProRoot MTA also significantly upregulated the expressions of ALP, DSPP, and OCN at day 10 as compared to the control. However, at day 14 , all gene expressions dropped to lower levels than that of the control $(p<0.05)$. For TheraCal LC, the gene expressions were all significantly lower than that of the control at all time intervals $(p<0.05)$; the only exception was the expression of OCN at day 14 , which was similar as the control $(p>0.05)$.

\subsection{Vital human tooth model}

In exposed pulps capped for 2 weeks, mineralized foci formation was detected for both TCS 50 and ProRoot MTA. However, for pulps capped with ThereCal LC, the pulp tissue revealed no sign of mineralized foci formation. Histological images of a representative tooth which was capped with TCS 50 for 2 weeks are presented in Fig. 7. H\&E staining showed the pulp tissue was well organized and mineralized foci were formed close to the exposure site (Fig. 7a). The mineralized foci varied in size and demonstrated an irregular shape with cellular components and pores entrapped in the matrix (Fig. 7b). Collagen-I expression was positive in the matrix of the pulp tissue (Fig. 7c), while negative within the matrix of mineralized foci (Fig. 7d). The expression of nestin, however, was negative for pulp tissue (Fig. 7e), while positive for the entrapped cellular components within mineralized foci (Fig. 7f). Immunostaining of collagen I and nestin for exposed pulps capped with ProRoot MTA showed a similar result.

After 4 weeks, mineralized foci were formed in all pulps capped with 3 pulp-capping agents; the only exception is a tooth which was capped with TheraCal LC. Similar as after 2 weeks, this capped pulp revealed no obvious mineralized foci formation. Histological images of 3 representative teeth capped with TCS 50, ProRoot MTA and TheraCal LC for 4 weeks are presented in Fig. 8. In general, the mineralized foci demonstrated a similar morphology as that after 2-week culturing. An overview of a TCS 50 capped tooth showed the spread of numerous mineralized foci underneath the exposure site (Fig. 8a). The mineralized foci still exhibited cells entrapped in the matrix (arrow in Fig. 8b). For the tooth which was capped with ProRoot MTA, mineralized foci were also presented at the area close to the exposure site (Fig. 8c). Cellular components were entrapped in the matrix of mineralized foci (arrow in Fig. 8d). In two out of three exposed pulps that were capped with TheraCal LC, mineralized foci were also formed (Fig. 8e). The mineralized foci morphology was similar to that induced by TCS 50 and ProRoot MTA (arrows in Fig. 8f). Immunostaining of collagen I and nestin showed a similar result in all generated mineralized foci. The expression of collagen I was weak within the matrix of mineralized foci, while nestin was intensively expressed in the residual cellular components within mineralized foci. 
For all exposed pulps without pulp-capping agent, pulp tissue without any signs of mineralization initiation or pulp healing was observed, regardless the culture period ( 2 or 4 weeks). Histological images of a representative tooth that was kept for 4 weeks revealed a normal morphology of the pulp tissue (Fig. 8g); a layer of necrotic pulp tissue was disclosed near the exposed surface, on top of a layer of connective tissue (Fig. 8h).

Bacteria were not detected at the cavity wall or within the pulp tissue, this for all the culture periods ( 2 or 4 weeks) and different pulp-capping agents.

\section{DISCUSSION}

Hypothesis (1) that TCS 50 showed no effect on the viability, proliferation, migration, and odontoblastic differentiation of HDPCs, was partially rejected. The effect of TCS 50 towards HDPCs appeared dose dependent. Undiluted TCS 50 extract displayed no adverse effect on cell viability, but it significantly inhibited cell proliferation and migration $(p<0.05)$. The $25 \%$ diluted TCS 50 extract showed no significant effect on cell viability, proliferation or migration $(p>0.05)$, and it significantly enhanced odontoblastic differentiation of HDPCs $(p<0.05)$. Hypothesis $(2)$ that the exposed pulps capped with TCS 50 did not display pulpal reaction after 2 or 4 weeks culturing, was fully rejected. Indeed, all exposed pulps capped with TCS 50 resulted in mineralized foci spread underneath the exposure site, this after both 2- and 4-week culturing. Hypothesis (3) that the effects of TCS 50 onto HDPCs and exposed human pulps were not favorable as compared to those of the reference hCSCs ProRoot MTA (Dentsply Sirona) and TheraCal LC (Bisco), was also rejected. Overall, the experimentally prepared hCSC TCS 50 performed similarly regarding its effect on HDPC viability, proliferation and odontoblastic differentiation as the reference hCSC ProRoot MTA. However, all diluted ProRoot MTA extracts significantly enhanced migration of HDPCs $(p<0.05)$, while TCS 50 dilutions exhibited no obvious effect. The pulpal reaction in human exposed pulps capped with TCS 50 was not different from that induced by ProRoot MTA, both after 2- and 4-week culture. On the contrary, the resin-based hCSC TheraCal LC induced a negative effect on HDPC viability, proliferation, and migration, indicating TheraCal LC'S cytotoxic effect on HDPCs. In line with the cellular findings, a less favorable pulpal reaction was observed in TheraCal LC capped pulps, as mineralized foci were formed at a later stage and in a lower percentage as compared to TCS 50 and ProRoot MTA capped pulps.

The cell-viability assessment confirmed the cytocompatibility of the resin-free hCSCS TCS 50 and ProRoot MTA versus that of the resin-based hCSC TheraCal LC and the ZnO-eugenol cement, which appeared cytotoxic. Regarding cell proliferation, HDPCs were seeded at a lower density to ensure proliferation over time. Undiluted TCS 50 extract solely slightly inhibited cell proliferation, with all diluted extracts possessing a similar favorable effect on cell proliferation as culture medium. Previous studies also claimed that tricalcium silicate, the main component of TCS 50 , stimulated proliferation of 
HDPCs [26] and the mouse fibroblast L929 cell line [27]. The biocompatibility of ProRoot MTA has been widely accepted $[12,28,29]$, and not surprisingly in this study, ProRoot MTA demonstrated also a cytocompatibility in contact with HDPCs. The cytotoxic effect of TheraCal LC to HDPCs is also in accordance with previous studies, which indicated a cytotoxicity of TheraCal LC towards human pulp fibroblasts [9,30], DPSCs [31], and stem cells isolated from human exfoliated primary teeth (SHEDs) [32]. The cytotoxicity of this resin-based material should most likely be attributed incomplete polymerization which leads to the leaching of unpolymerized resin monomers $[33,34]$.

The in-vitro wound-healing assay revealed that the TCS 50 undiluted extracts prevented migration of DPSCs, while the diluted extracts exhibited no obvious effect. Further evaluation of the expression of chemokines, which are involved in cell recruitment, is needed to better understand the mechanism behind this phenomenon. The effect of ProRoot MTA was also dose-dependent; all diluted extracts largely enhanced the cell migration, while the undiluted extract generated a negative effect. This finding is in disagreement with a previous study that reported that an MTA extract of a similar concentration as the current undiluted extract significantly promoted cell migration [12]. This inconsistency in data could be ascribed to different methodologies applied to prepare the cement extracts. Unlike the current study, in which cells were immediately exposed to freshly mixed cement extracts, the previous study stored cement disks for 3 days prior to immersion in the medium, while the extract was also filtered and collected after 3-day immersion [12]. For TheraCal LC, both the undiluted and diluted extracts showed an adverse effect on cell migration, which should, at least in part, be attributed to its cytotoxic effect on HDPCs. A previous study also revealed that TheraCal LC decreased migration of SHEDs, even when the cement extract was prepared using cement disks that were incubated for 2 days to achieve complete setting and the extract was filtered to remove components that had been released by the cement [32].

To evaluate cell odontoblastic differentiation, the associated genes ALP, DSPP, and OCN were selected as markers. ALP is known to be present in the early phase of differentiation and is involved in the mineralization process via the handling of calcium and phosphate $[35,36]$. DSPP is produced by terminally differentiated odontoblasts; it is widely accepted as an odontoblast-specific gene that modulates dentin mineralization [36,37]. OCN is synthesized by mature osteoblasts, odontoblasts and cementoblasts; it is also considered as a late cell-differentiation marker [37,38]. Previous research showed that calcium-silicate bioceramics could significantly enhance osteogenic differentiation, therefore promoting bone regeneration $[39,40]$. Here, the result revealed that the $25 \%$ diluted TCS 50 extract enhanced odontoblastic differentiation of DPSCs via up-regulation of expression of the early differentiation marker ALP at day 10 as well as of the lateral differentiation markers DSPP and OCN at day 10 and 14 . A previous study also reported that tricalcium silicate at a concentration of $5 \mathrm{mg} / \mathrm{mL}$ amplified the expression of ALP, DSPP, and OCN by HDPCs [26]. ProRoot MTA also promoted cell 
odontoblastic differentiation by increasing the expression of ALP, DSPP and OCN at day 10. This result is consistent with a previous study, which indicated that the expression of these three markers increased in HDPCs that were exposed to ProRoot MTA for 7 days [41]. TheraCal LC extract, however, significantly downregulated the expression of ALP, DSPP and OCN, as compared to odontogenic differentiation medium, this at day 4, 10 and 14. Only at day 14, the OCN expressions in HDPCs that were exposed to TheraCal LC reached a similar level as that of the odontogenic differentiation medium. This confirmed the less favorable effect of TheraCal LC on cell odontoblastic differentiation. A recent study also disclosed that the expression of osteogenesis-related genes, including ALP, bone sialoprotein (BSP), DSPP, dentin matrix protein-1 (DMP-1) and OCN, in hDPSCs that were exposed to TheraCal LC extract, were not significantly different from that in untreated hDPSCs which were exposed to culture medium [31].

Altogether, the effect of pulp-capping agents on viability, proliferation, migration, and odontoblastic differentiation are all dose-dependent; therefore, comparison of the results from different studies can only be done by selecting a standardized cement-extract preparation method. Unfortunately, the absence of a clinically relevant standard has led to the application of various cement-extract preparation methods in literature. In the current study, extracts of freshly mixed and unset cement disks were directly collected without filtering, this in order to evaluate the cement effect in a more clinically relevant condition [13]. Furthermore, the immersion medium for the cement disks was refreshed to simulate the dynamic oral environment. Additionally, the ratio of the surface area of the cement disk to the volume of cement extract was $127.8 \mathrm{~mm} / \mathrm{mL}$, which approximates the value recommended in the ISO standard 10993-5:2009 (150-600 mm²/mL). Differently, the majority of previous studies tested cements in a set state without refreshing the immersion medium, while the ratio of the surface area of the cement disk to the volume of cement extract was either not mentioned or varied from 4.5 to $19.0 \mathrm{~mm}^{2} / \mathrm{mL}[12,36,37]$. In this respect, the extracts employed in this study may have contained more leachable components from the cements and may also be more concentrated; this may explain the differences in results found in this study versus the previous findings.

On the basis of the results of this study, TCS 50 was proven to be a promising hCSC formulation. Noteworthy is that the effectiveness of a pulp-capping agent should not be predicted solely on basis of the cellular responses encountered in vitro. The employed monolayer cell-culture model has limitations, as there is for instance the lack of cell-to-cell and cell-to-extracellular matrix interaction [42]. To further prove the pulp-repair potential of TCS 50 , a vital human tooth model was used in this study. The result was consistently positive, as TCS 50 induced mineralized foci formation in mechanically exposed pulps after 2 and 4 weeks. These mineralized foci varied in sizes and demonstrated an immature appearance with the presence of cells entrapped in the matrix. Immunochemistry revealed that nestin was positively expressed in the sequestered cells within 
mineralized foci, suggesting that the formed mineralized foci corresponded to an initial form of reparative dentin formation [24] as the expression of this intermediate filament protein was shown as specific and characteristic of the secretory human odontoblast [43]. ProRoot MTA also generated mineralized foci formation of a similar morphology, confirming the results from a previous study [24]. TheraCal LC induced mineralized foci formation in exposed pulps at a lower speed and in a lower percentage; this may be due to the incomplete hydration of the material. A previous study investigated the hydration of TheraCal LC using the same vital human tooth model; it disclosed a limited moisture diffusion within the material, which had resulted in incomplete hydration and lower release of calcium ions [44]. The calcium concentration in the cellular environment is very crucial, as it is expected to invoke migration of stem cells and differentiation to odontoblast-like cells, two essential processes involved in pulpal repair $[45,46]$.

The pulpal response obtained in vital human tooth model correlates with the features of pulpal reaction monitored in a minipig animal model [21]. In a recent study, we evaluated the pulpal repair potential of the abovementioned three pulp-capping agents when applied onto exposed minipig pulps [21]. For TCS 50, the formation of complete reparative dentin with the presence of dental tubular structures was observed in all pulps after 70 days. ProRoot MTA generated a similar response, while TheraCal LC induced a less favorable reaction, this in terms of initial inflammation and reparative dentin formation. While the vital human tooth model employed in this study generated clear indications on pulpal repair potential, the preclinical laboratory model has inherent limitations, as it solely revealed histological features of the pulp tissues at a particular time; the transformation process of odontoblasts could not be monitored. In addition, it can only record pulpal reaction at an early stage, while the lack of circulation and oxygen exchange in the pulp environment should be considered in the interpretation of the results.

\section{CONCLUSION}

On basis of the evidence derived from an in-vitro cellular study and using vital human tooth model, it is concluded that the experimental hCSC TCS 50 promoted odontoblastic differentiation of HDPCs and initiated pulp healing. Therefore, TCS 50 may serve as a promising pulp-capping agent.

\section{ACKNOWLEDGEMENTS}

This work was supported by the governmental grant G089315N of the Research Foundation - Flanders. We would like to acknowledge the Department of Oral and Maxillofacial Surgery for the collection of extracted human third molars and Mr. Wilfried Versin from the Department of Imaging and Pathology for assisting with the histological processing. We would also like to thank the dental manufacturers 
Bisco, Dentsply Sirona and GC for providing us the commercial materials. The authors declare that there is no conflict of interest related to this study.

\section{REFERENCES}

[1] P.N.R. Nair, H.F. Duncan, T.R. Pitt Ford, H.U. Luder, Histological, ultrastructural and quantitative investigations on the response of healthy human pulps to experimental capping with mineral trioxide aggregate: A randomized controlled trial, Int. Endod. J. (2009). https://doi.org/10.1111/j.1365-2591.2009.01558.x.

[2] T.J. Hilton, J.L. Ferracane, L. Mancl, Comparison of $\mathrm{CaOH}$ with MTA for Direct Pulp Capping: A PBRN Randomized Clinical Trial, J. Dent. Res. (2013). https://doi.org/10.1177/0022034513484336.

[3] M. Parirokh, M. Torabinejad, Mineral Trioxide Aggregate: A Comprehensive Literature ReviewPart III: Clinical Applications, Drawbacks, and Mechanism of Action, J. Endod. (2010). https://doi.org/10.1016/j.joen.2009.09.009.

[4] B.R. Johnson, Considerations in the selection of a root-end filling material, Oral Surg. Oral Med. Oral Pathol. Oral Radiol. Endod. (1999). https://doi.org/10.1016/S1079-2104(99)702374.

[5] M. Torabinejad, C.U. Hong, F. McDonald, T.R. Pitt Ford, Physical and chemical properties of a new root-end filling material, J. Endod. (1995). https://doi.org/10.1016/S00992399(06)80967-2.

[6] M. Schembri, G. Peplow, J. Camilleri, Analyses of heavy metals in mineral trioxide aggregate and Portland cement, J. Endod. (2010). https://doi.org/10.1016/j.joen.2010.02.011.

[7] M. Maroto, E. Barbería, V. Vera, F. García-Godoy, Dentin bridge formation after white mineral trioxide aggregate (white MTA) pulpotomies in primary molars, Am. J. Dent. (2006).

[8] X. Li, K. Yoshihara, J. De Munck, S. Cokic, P. Pongprueksa, E. Putzeys, M. Pedano, Z. Chen, K. Van Landuyt, B. Van Meerbeek, Modified tricalcium silicate cement formulations with added zirconium oxide, Clin. Oral Investig. 21 (2017) 895-905. https://doi.org/10.1007/s00784-0161843-y.

[9] U. Schröder, Effects of Calcium Hydroxide-containing Pulp-capping Agents on Pulp Cell Migration, Proliferation, and Differentiation, J. Dent. Res. 64 (1985) 541-8. https://doi.org/10.1177/002203458506400407.

[10] J.L. Sanz, F.J. Rodríguez-Lozano, C. Llena, S. Sauro, L. Forner, Bioactivity of bioceramic materials used in the dentin-pulp complex therapy: A systematic review, Materials (Basel). (2019). https://doi.org/10.3390/ma12071015.

[11] C.M. Corral Nuñez, H.J. Bosomworth, C. Field, J.M. Whitworth, R.A. Valentine, Biodentine and 
Mineral Trioxide Aggregate Induce Similar Cellular Responses in a Fibroblast Cell Line, J. Endod. (2014). https://doi.org/10.1016/j.joen.2013.11.006.

[12] L. Zhu, J. Yang, J. Zhang, B. Peng, A comparative study of bioaggregate and ProRoot MTA on adhesion, migration, and attachment of human dental pulp cells, J. Endod. (2014). https://doi.org/10.1016/j.joen.2013.12.028.

[13] M.S. Pedano, X. Li, S. Li, Z. Sun, S.M. Cokic, E. Putzeys, K. Yoshihara, Y. Yoshida, Z. Chen, K. Van Landuyt, B. Van Meerbeek, Freshly-mixed and setting calcium-silicate cements stimulate human dental pulp cells, Dent. Mater. (2018) 2-13. https://doi.org/10.1016/j.dental.2018.02.005.

[14] M. Kuratate, K. Yoshiba, Y. Shigetani, N. Yoshiba, H. Ohshima, T. Okiji, Immunohistochemical Analysis of Nestin, Osteopontin, and Proliferating Cells in the Reparative Process of Exposed Dental Pulp Capped with Mineral Trioxide Aggregate, J. Endod. (2008). https://doi.org/10.1016/j.joen.2008.03.021.

[15] X. V. Tran, C. Gorin, C. Willig, B. Baroukh, B. Pellat, F. Decup, S. Opsahl Vital, C. Chaussain, T. Boukpessi, Effect of a calcium-silicate-based restorative cement on pulp repair, J. Dent. Res. (2012). https://doi.org/10.1177/0022034512460833.

[16] H. Sazak, M. Günday, C. Alatli, Effect of calcium hydroxide and combinations of ledermix and calcium hydroxide on inflamed pulp in dog teeth, J. Endod. (1996).

https://doi.org/10.1016/S0099-2399(96)80074-4.

[17] E. Koliniotou-Koumpia, D. Tziafas, Pulpal responses following direct pulp capping of healthy dog teeth with dentine adhesive systems, J. Dent. (2005). https://doi.org/10.1016/j.jdent.2004.12.007.

[18] S.H. Dickens, G.M. Flaim, G.E. Schumacher, F.C. Eichmiller, D.R. Schafer, R.B. Rutherford, Preclinical effectiveness of a novel pulp capping material, J. Endod. (2010). https://doi.org/10.1016/j.joen.2010.02.021.

[19] M. Cvek, L. Granath, P. Cleaton-Jones, J. Austin, Hard Tissue Barrier Formation in Pulpotomized Monkey Teeth Capped with Cyanoacrylate or Calcium Hydroxide for 10 and 60 Minutes, J. Dent. Res. (1987). https://doi.org/10.1177/00220345870660061501.

[20] C. Tziafa, E. Koliniotou-Koumpia, S. Papadimitriou, D. Tziafas, Dentinogenic responses after direct pulp capping of miniature swine teeth with Biodentine, J. Endod. (2014). https://doi.org/10.1016/j.joen.2014.07.021.

[21] X. Li, M.S. Pedano, B. Camargo, E. Hauben, S. De Vleeschauwer, Z. Chen, J. De Munck, K. Vandamme, K. Van Landuyt, B. Van Meerbeek, Experimental tricalcium silicate cement induces reparative dentinogenesis, Dent. Mater. (2018) 1-14. https://doi.org/10.1016/j.dental.2018.06.016. 
[22] T. Dammaschke, Rat molar teeth as a study model for direct pulp capping research in dentistry, Lab. Anim. (2010). https://doi.org/10.1258/la.2009.008120.

[23] A. Watts, R.C. Paterson, A comparison of pulp responses to two different materials in the dog and the rat, Oral Surgery, Oral Med. Oral Pathol. (1981). https://doi.org/10.1016/00304220(81)90085-2.

[24] O. Téclès, P. Laurent, V. Aubut, I. About, Human tooth culture: A study model for reparative dentinogenesis and direct pulp capping materials biocompatibility, J. Biomed. Mater. Res. Part B Appl. Biomater. (2008). https://doi.org/10.1002/jbm.b.30933.

[25] P. Laurent, J. Camps, I. About, Biodentine induces TGF- $\beta 1$ release from human pulp cells and early dental pulp mineralization, Int. Endod. J. (2012). https://doi.org/10.1111/j.13652591.2011.01995.x.

[26] W. Peng, W. Liu, W. Zhai, L. Jiang, L. Li, J. Chang, Y. Zhu, Effect of tricalcium silicate on the proliferation and odontogenic differentiation of human dental pulp cells, J. Endod. (2011). https://doi.org/10.1016/j.joen.2011.05.035.

[27] W. Zhao, J. Wang, W. Zhai, Z. Wang, J. Chang, The self-setting properties and in vitro bioactivity of tricalcium silicate, Biomaterials. (2005). https://doi.org/10.1016/j.biomaterials.2005.04.025.

[28] T. Takita, M. Hayashi, O. Takeichi, B. Ogiso, N. Suzuki, K. Otsuka, K. Ito, Effect of mineral trioxide aggregate on proliferation of cultured human dental pulp cells, Int. Endod. J. (2006). https://doi.org/10.1111/j.1365-2591.2006.01097.x.

[29] L. Zhu, J. Yang, J. Zhang, D. Lei, L. Xiao, X. Cheng, Y. Lin, B. Peng, In vitro and in vivo evaluation of a nanoparticulate bioceramic paste for dental pulp repair, Acta Biomater. (2014). https://doi.org/10.1016/j.actbio.2014.08.014.

[30] C. Jeanneau, P. Laurent, C. Rombouts, T. Giraud, I. About, Light-cured Tricalcium Silicate Toxicity to the Dental Pulp, J. Endod. (2017). https://doi.org/10.1016/j.joen.2017.07.010.

[31] E.A. Bortoluzzi, L.N. Niu, C.D. Palani, A.R. El-Awady, B.D. Hammond, D.D. Pei, F.C. Tian, C.W. Cutler, D.H. Pashley, F.R. Tay, Cytotoxicity and osteogenic potential of silicate calcium cements as potential protective materials for pulpal revascularization, Dent. Mater. (2015). https://doi.org/10.1016/j.dental.2015.09.020.

[32] M. Collado-González, D. García-Bernal, R.E. Oñate-Sánchez, P.S. Ortolani-Seltenerich, T. Álvarez-Muro, A. Lozano, L. Forner, C. Llena, J.M. Moraleda, F.J. Rodríguez-Lozano, Cytotoxicity and bioactivity of various pulpotomy materials on stem cells from human exfoliated primary teeth, Int. Endod. J. (2017). https://doi.org/10.1111/iej.12751.

[33] W.F. Caughman, G.B. Caughman, R.A. Shiflett, F. Rueggeberg, G.S. Schuster, Correlation of cytotoxicity, filler loading and curing time of dental composites, Biomaterials. (1991). 
https://doi.org/10.1016/0142-9612(91)90022-3.

[34] L. Stanislawski, X. Daniau, A. Lautié, M. Goldberg, Factors responsible for pulp cell cytotoxicity induced by resin-modified glass ionomer cements, J. Biomed. Mater. Res. (1999). https://doi.org/10.1002/(SICI)1097-4636(1999)48:3<277::AID-JBM11>3.0.CO;2-T.

[35] S.S. Goseki M, Oida S, Nifuji A, Properties of alkaline phosphatase of the human dental pulp, J Dent Res. 69 (1990) 909-12.

[36] C. Bègue-Kirn, P.H. Krebsbach, J.D. Bartlett, W.T. Butler, Dentin sialoprotein, dentin phosphoprotein, enamelysin and ameloblastin: Tooth-specific molecules that are distinctively expressed during murine dental differentiation, Eur. J. Oral Sci. (1998). https://doi.org/10.1046/j.0909-8836.1998.eos106510.x.

[37] P. Papagerakis, A. Berdal, M. Mesbah, M. Peuchmaur, L. Malaval, J. Nydegger, J. Simmer, M. Macdougall, Investigation of osteocalcin, osteonectin, and dentin sialophosphoprotein in developing human teeth, Bone. (2002). https://doi.org/10.1016/S8756-3282(01)00683-4.

[38] X. Wei, J. Ling, L. Wu, L. Liu, Y. Xiao, Expression of Mineralization Markers in Dental Pulp Cells, J. Endod. (2007). https://doi.org/10.1016/j.joen.2007.02.009.

[39] H. Li, K. Xue, N. Kong, K. Liu, J. Chang, Silicate bioceramics enhanced vascularization and osteogenesis through stimulating interactions between endothelia cells and bone marrow stromal cells, Biomaterials. (2014). https://doi.org/10.1016/j.biomaterials.2014.01.039.

[40] V. Bunpetch, X. Zhang, T. Li, J. Lin, E.P. Maswikiti, Y. Wu, D. Cai, J. Li, S. Zhang, C. Wu, H. Ouyang, Silicate-based bioceramic scaffolds for dual-lineage regeneration of osteochondral defect, Biomaterials. (2019). https://doi.org/10.1016/j.biomaterials.2018.11.025.

[41] S.W. Chang, S.Y. Lee, K.Y. Kum, E.C. Kim, Effects of ProRoot MTA, bioaggregate, and micromega MTA on odontoblastic differentiation in human dental pulp cells, J. Endod. (2014). https://doi.org/10.1016/j.joen.2013.09.036.

[42] F. Pampaloni, E.G. Reynaud, E.H.K. Stelzer, The third dimension bridges the gap between cell culture and live tissue, Nat. Rev. Mol. Cell Biol. (2007). https://doi.org/10.1038/nrm2236.

[43] I. About, D. Laurent-Maquin, U. Lendahl, T.A. Mitsiadis, Nestin expression in embryonic and adult human teeth under normal and pathological conditions, Am. J. Pathol. (2000). https://doi.org/10.1016/S0002-9440(10)64539-7.

[44] J. Camilleri, P. Laurent, I. About, Hydration of biodentine, theracal LC, and a prototype tricalcium silicate-based dentin replacement material after pulp capping in entire tooth cultures, J. Endod. (2014). https://doi.org/10.1016/j.joen.2014.06.018.

[45] K.E. Kardos TB, Hunter AR, Hanlin SM, Odontoblast differentiation: a response to environmental calcium?, Endod Dent Traumatol. 14 (1998) 105-11.

[46] G.B. Adams, K.T. Chabner, I.R. Alley, D.P. Olson, Z.M. Szczepiorkowski, M.C. Poznansky, C.H. 
Kos, M.R. Pollak, E.M. Brown, D.T. Scadden, Stem cell engraftment at the endosteal niche is specified by the calcium-sensing receptor, Nature. (2006).

https://doi.org/10.1038/nature04247. 


\section{TABLES}

Table 1. List of the primers used for RT-PCR

\begin{tabular}{llc}
\hline Primer & \multicolumn{1}{c}{ Sequence (5'-3') } & Size (bp) \\
\hline Alkaline phosphatase (ALP) & $\begin{array}{l}\text { F: CCA CGT CTT CAC ATT TGG TG } \\
\text { R: GCA GTG AAG GGC TTC TTG TC }\end{array}$ & 96 \\
Dentin sialophosphoprotein (DSPP) & F: TTC CGA TGG GAG TCC TAG TG & \multirow{2}{*}{144} \\
& R: TCT TCT TTC CCA TGG TCC TG & \\
Osteocalcin (OCN) & F: GAC TGT GAC GAG TTG GCT GA & 83 \\
& R: AGC AGA GCG ACA CCC TAG AC & \\
Glyceraldehyde 3-phosphate dehydrogenase & F: CGA CCA CTT TGT CAA GCT CA & 59 \\
(GADPH) & R: CCC TGT TGC TGT AGC CAA AT & \\
\hline
\end{tabular}




\section{FIGURE LEGENDS}

Fig. 1 Vital human tooth model. Healthy human immature third molars with one- to two-third root formations as well as open apices were selected (a). A pulp exposure with a diameter of $1.0 \mathrm{~mm}$ was prepared at the occlusal surface (b). The pulp exposure was sealed with a pulp-capping agent applied in a thickness of approximately $2 \mathrm{~mm}$ (c). The crown was fixed to a wire and the tooth was suspended in the culture medium, which permitted diffusion of medium through the open apex (d).

Fig. 2 Relative formazan formation of HDPCs that were exposed to 1-day undiluted (100\%) and diluted (50\%, 25\% and 10\%) cement eluates of TCS 50 (experimental), ProRoot MTA (Dentsply Sirona), TheraCal LC (Bisco), and ZnO-eugenol cement (Alganol, Kemdent). The bar heights represent the mean values, and the line extensions represent standard deviations. For each concentration of cement eluate, cements labeled with the same capital letter are not significantly different $(p>0.05)$. For each cement, cement eluate concentrations labeled with the same lowercase letter are not significantly different $(p>0.05)$.

Fig. 3 Relative formazan formation of HDPCs that were exposed to TCS 50 (experimental), ProRoot MTA (Dentsply Sirona), TheraCal LC (Bisco), and ZnO-eugenol cement (Alganol, Kemdent), as undiluted $100 \%$ in (a), $50 \%$ diluted eluate in (b), $25 \%$ in (c), and $10 \%$ in (d), as well as the culture medium (control) for 1, 4 and 7 days. The bar heights represent the mean values, and the line extensions represent standard deviations. For each cement eluate concentration at the same time interval, cements labeled with the same capital letter are not significantly different $(p>0.05)$. For the same cement of the same eluate concentration, the evaluation time intervals labeled with the same lowercase letter are not significantly different $(p>0.05)$.

Fig. 4 Number of migrated HDPCs exposed to undiluted (100\%) and diluted (50\%, 25\% and $10 \%)$ eluates of TCS 50 (experimental), ProRoot MTA (Dentsply Sirona), TheraCal LC (Bisco), and the culture medium (control). In each box plot, the vertical line represents the full range of the number of migrated cells; the horizontal line in the box represents the mean value. For each cement eluate concentration, cements labeled with the same capital letter are not significantly different $(p>0.05)$. For each cement, cement eluate concentrations labeled with the same lowercase letter are not significantly different $(p>0.05)$.

Fig. 5 Representative photomicrographs of the scratched HDPC area that were exposed to $25 \%$ diluted eluates of TCS 50 (experimental) in (a,b), ProRoot MTA (Dentsply Sirona) in (c,d), TheraCal LC (Bisco) 
in $(e, f)$ and the culture-medium control in $(g, h)$. The photomicrographs were taken at 0 and $24 \mathrm{hrs}$ post-wound induction. The borders of the scratched area were labeled with two black lines. The number of cells, which migrated to the scratched area after $24 \mathrm{hrs}$, was counted independently by two researchers.

Fig. 6 Relative expression of ALP (a), DSPP (b), and OCN (c) by HDPCs that were exposed to $25 \%$ diluted eluates of TCS 50 (experimental), ProRoot MTA (Dentsply Sirona), TheraCal LC (Bisco), and odontogenic differentiation medium (control) for 4, 10 and 14 days. The bar heights represent the mean values, and the line extensions represent standard deviations. For each gene at the same time interval, cements labeled with the same capital letter are not significantly different $(p>0.05)$.

Fig. 7 Histological photomicrographs of a representative tooth that was capped with TCS 50 for 2 weeks. H\&E staining showed that the capped pulp tissue remained well organized with mineralized foci scattered underneath the exposure site (a). At higher magnification, entrapped cellular components and pores (arrow in b) were detected in the mineralized foci ( $b$ : higher magnification of the rectangular box in a). Immunostaining for collagen I revealed strong and uniformly intense staining for pulp tissue, while weak reactivity in dentin (c). In the matrix of the mineralized foci (arrow in d), staining was also weak ( $d$ : higher magnification of the rectangular box in c). On the contrary, nestin immunostaining was weak in the matrix of the pulp tissue (e), while in the entrapped cellular components within the mineralized foci, the staining was strong ( $\mathrm{f}$ : higher magnification of the rectangular box in e). $\mathrm{E}$ : exposure site; D: dentin; P: pulp tissue.

Fig. 8 Histological H\&E stained photomicrograps of four representative teeth that were capped for 4 weeks with TCS 50 in (a,b), ProRoot MTA (Dentsply Sirona) in (c,d), TheraCal LC (Bisco) in (e,f); no pulpcapping agent was applied as control in (g,h). An overview photomicrograph of the tooth capped with TCS 50 revealed remnants of TCS 50 located at the exposure site (a). Beneath the exposure site, mineralized foci in various sizes could be observed (arrow in a). At higher magnification, the mineralized foci with entrapped cells (arrow in b: higher magnification of the rectangular box in a). An overview of a tooth capped with ProRoot MTA showed a similar morphology; within the well-organized pulp tissue, various mineralized foci (arrow in c) were formed underneath the exposure site (c). At higher magnification, entrapped cells and pores (arrow in $d$ ) were also present ( $d$ : higher magnification of the rectangular box in c). An overview photomicrograph of a tooth capped with TheraCal LC also indicated the formation of mineralized foci underneath the exposure site (e); cells were entrapped in the mineralized foci (arrows in f: higher magnification of the rectangular box in e). An overview photomicrograph of an exposed tooth without pulp-capping agent revealed a normal morphology of 
pulp tissue (g). At higher magnification, a superficial layer of necrotic pulp tissue was observed underneath the exposure site; beneath this necrotic layer, connective tissue was detected (h: higher magnification of the rectangular box in g). E: exposure site; D: dentin; P: pulp tissue. 
Fig. 1
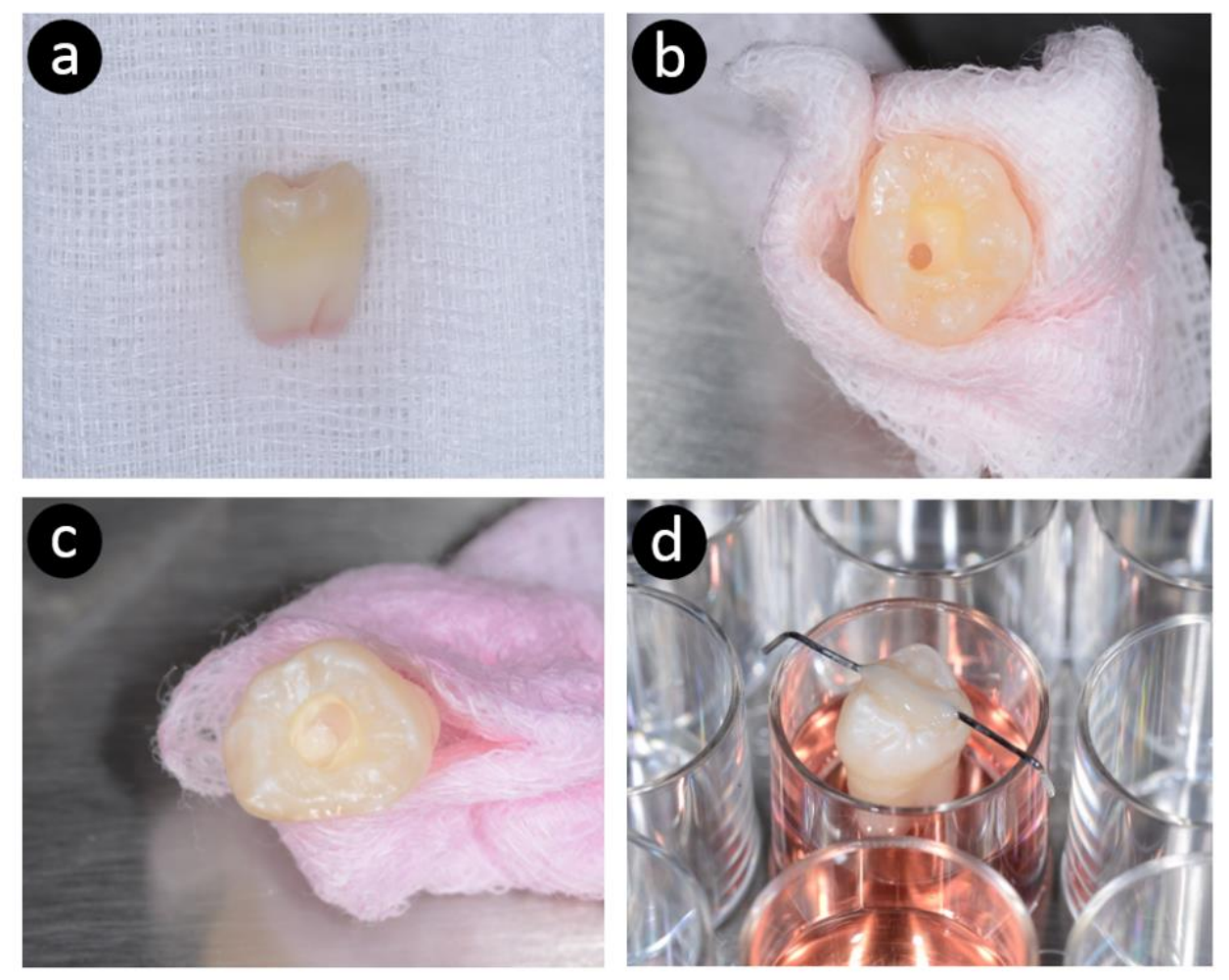

Fig. 2

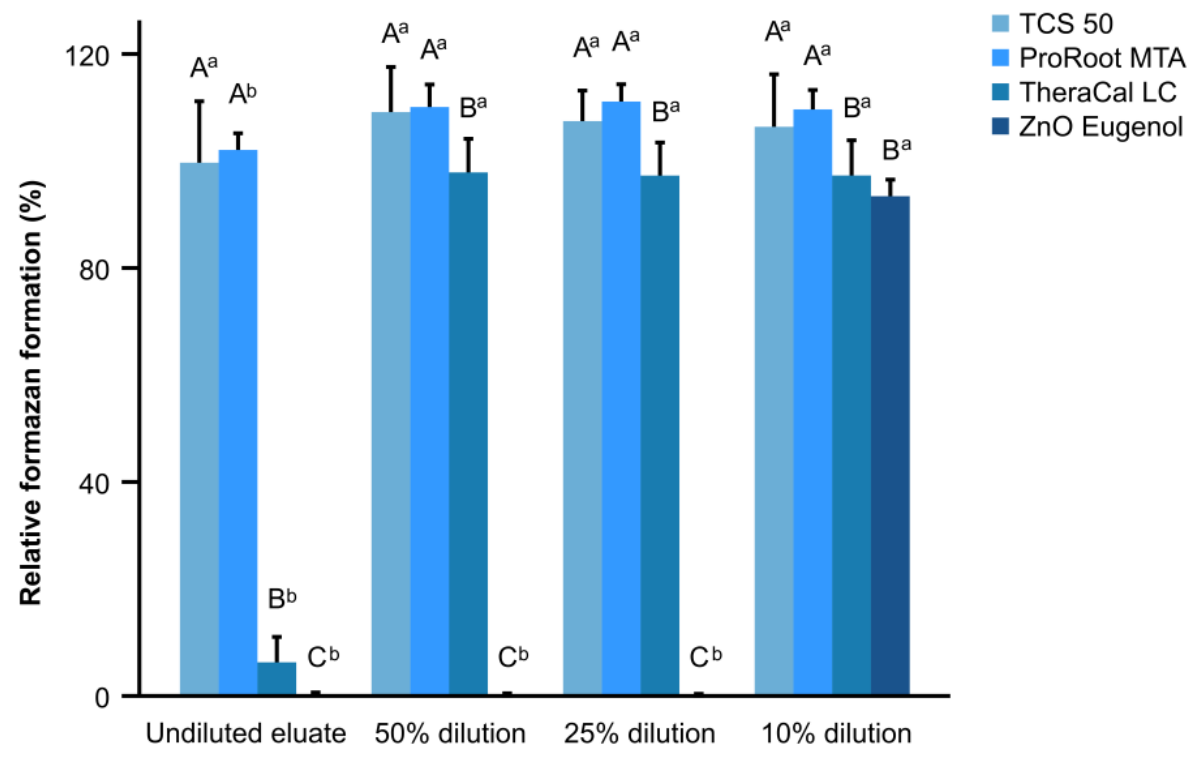


Fig. 3
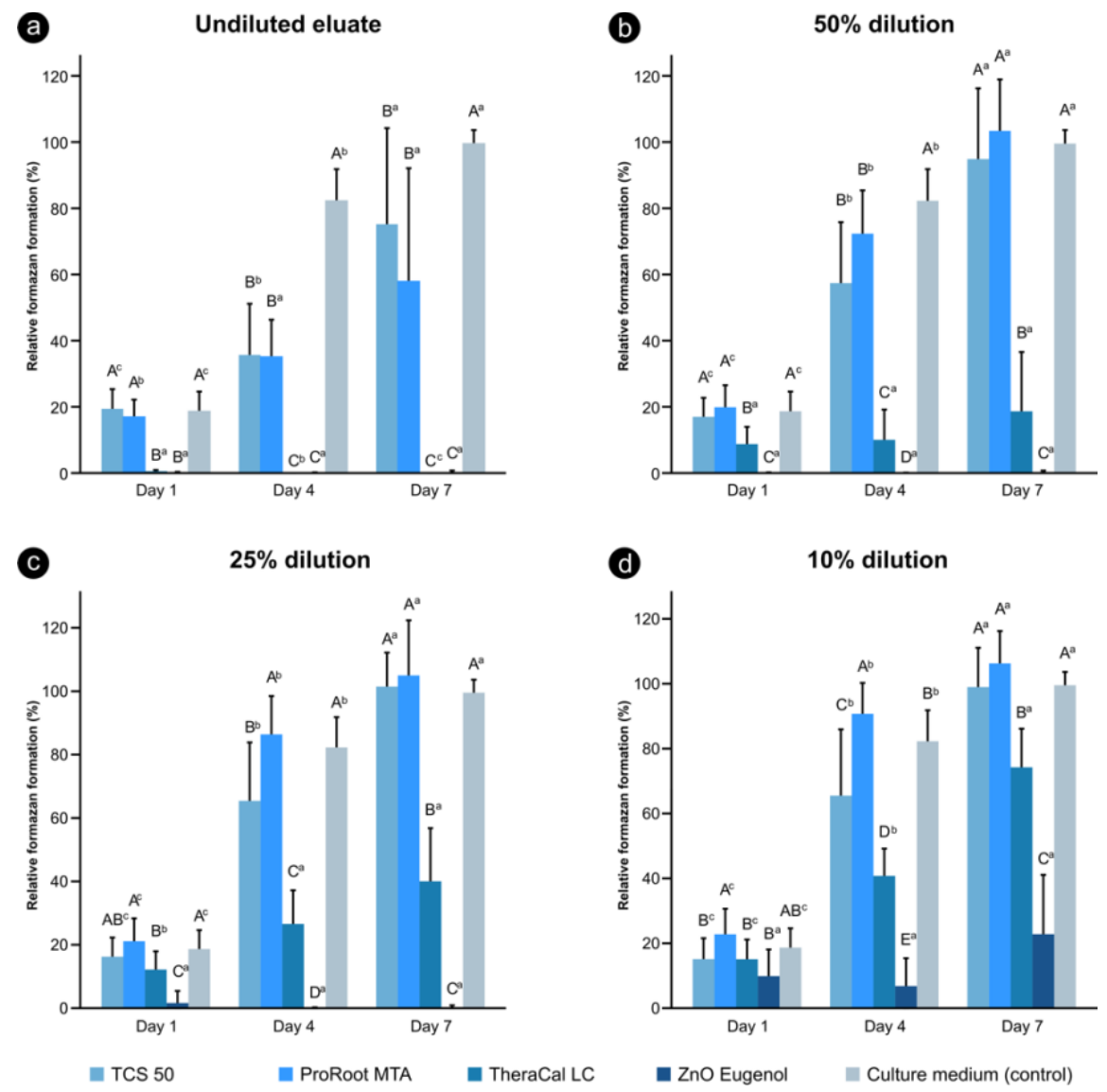

Fig. 4

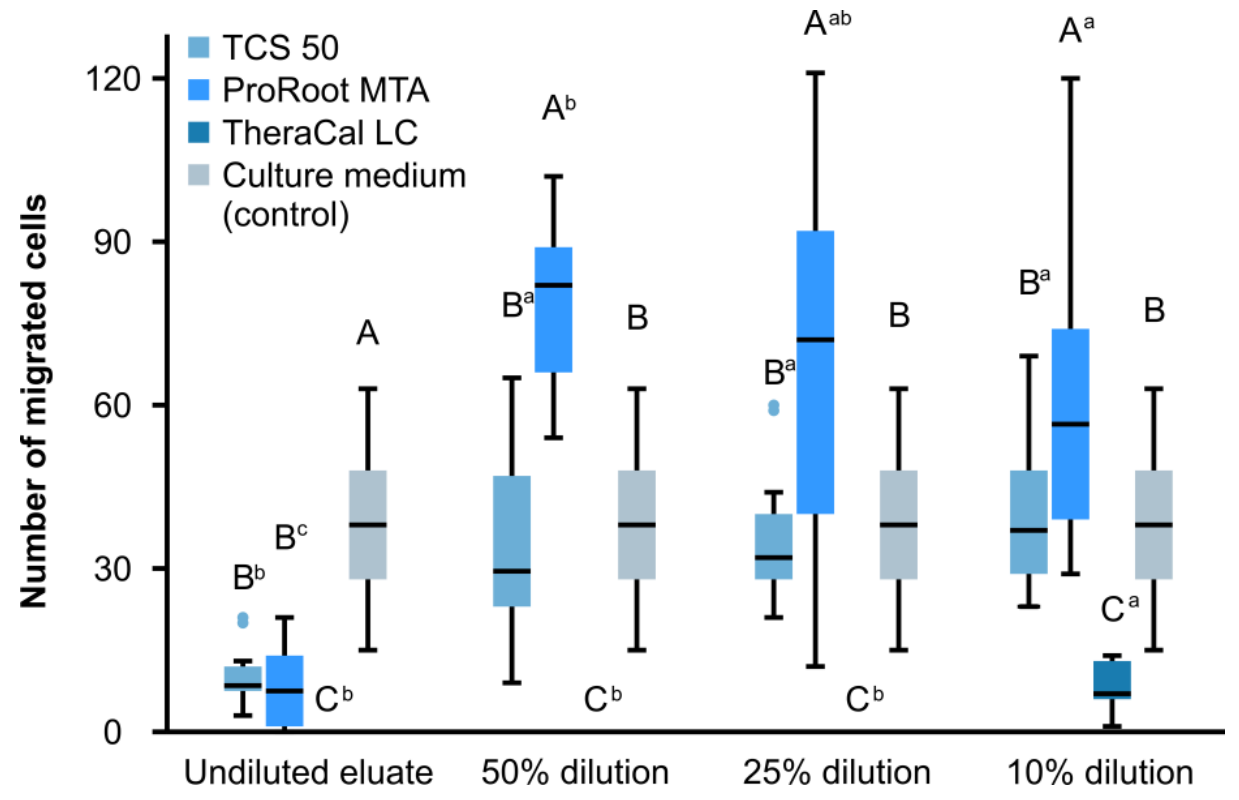


Fig. 5
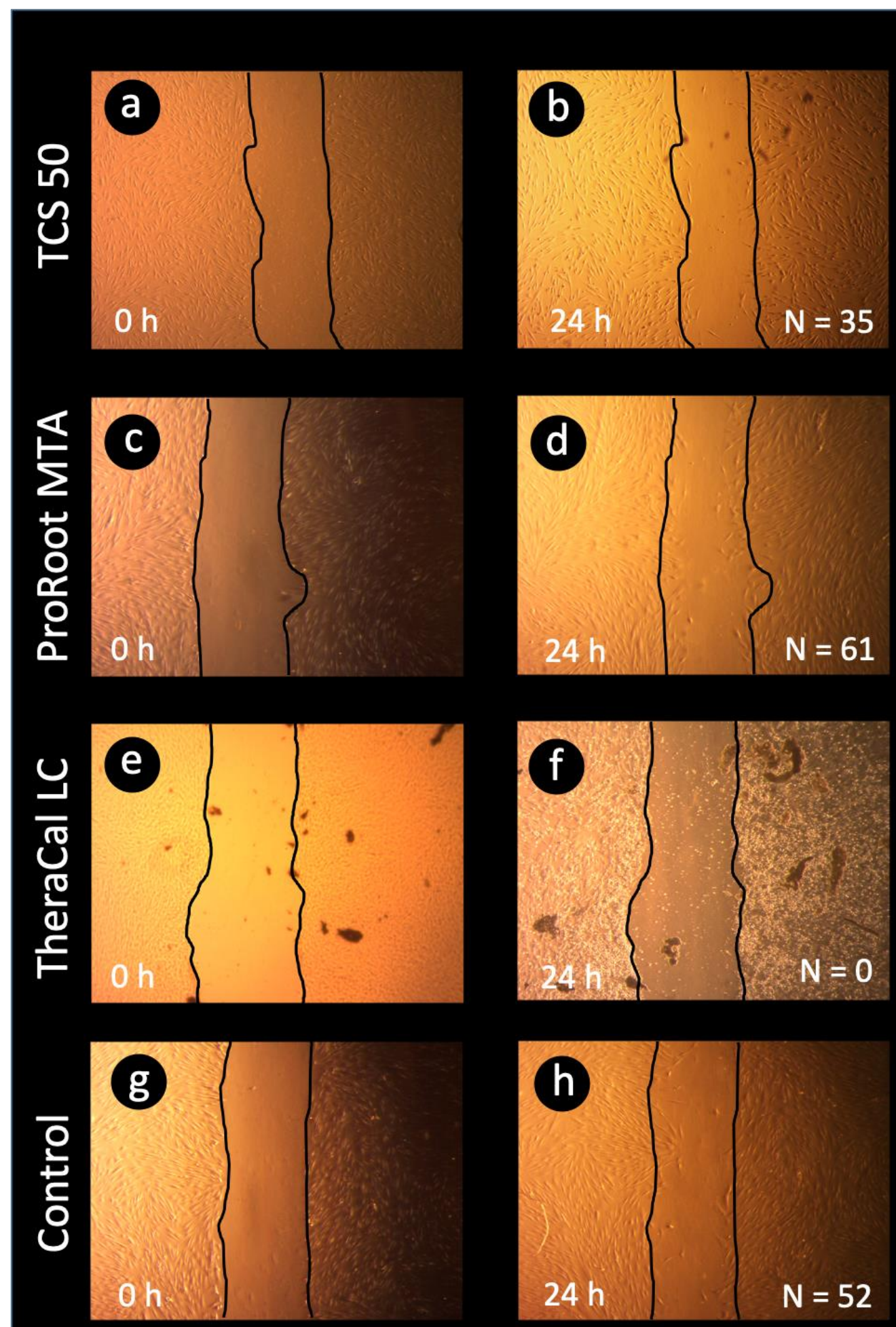
Fig. 6
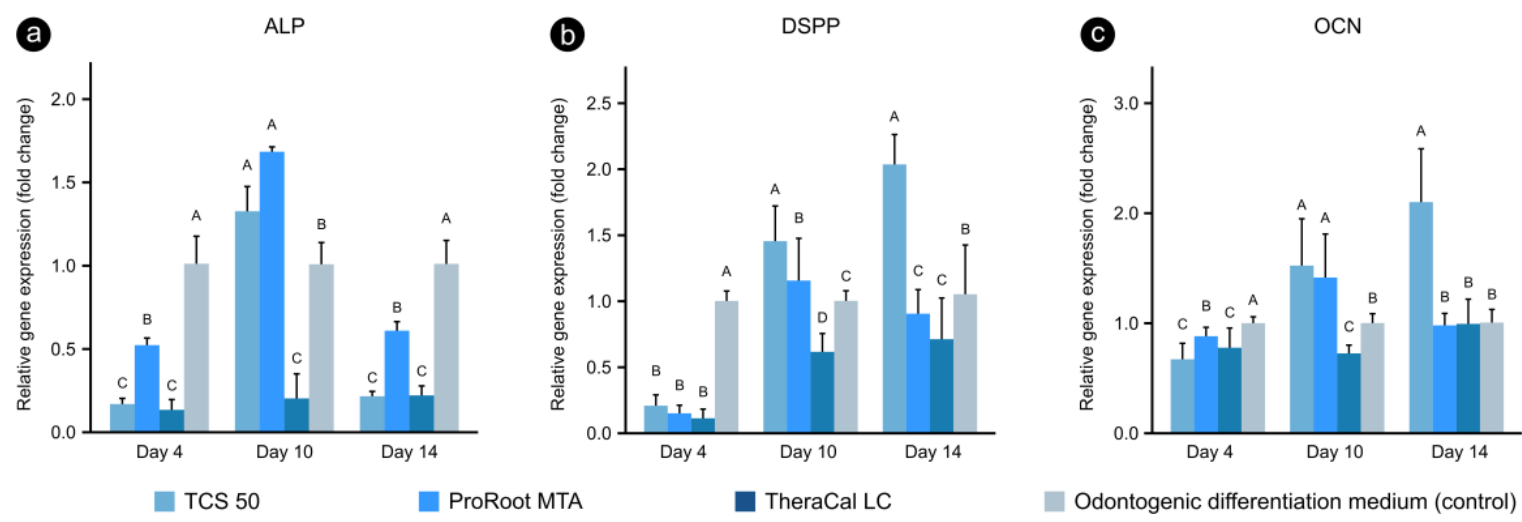

Fig. 7
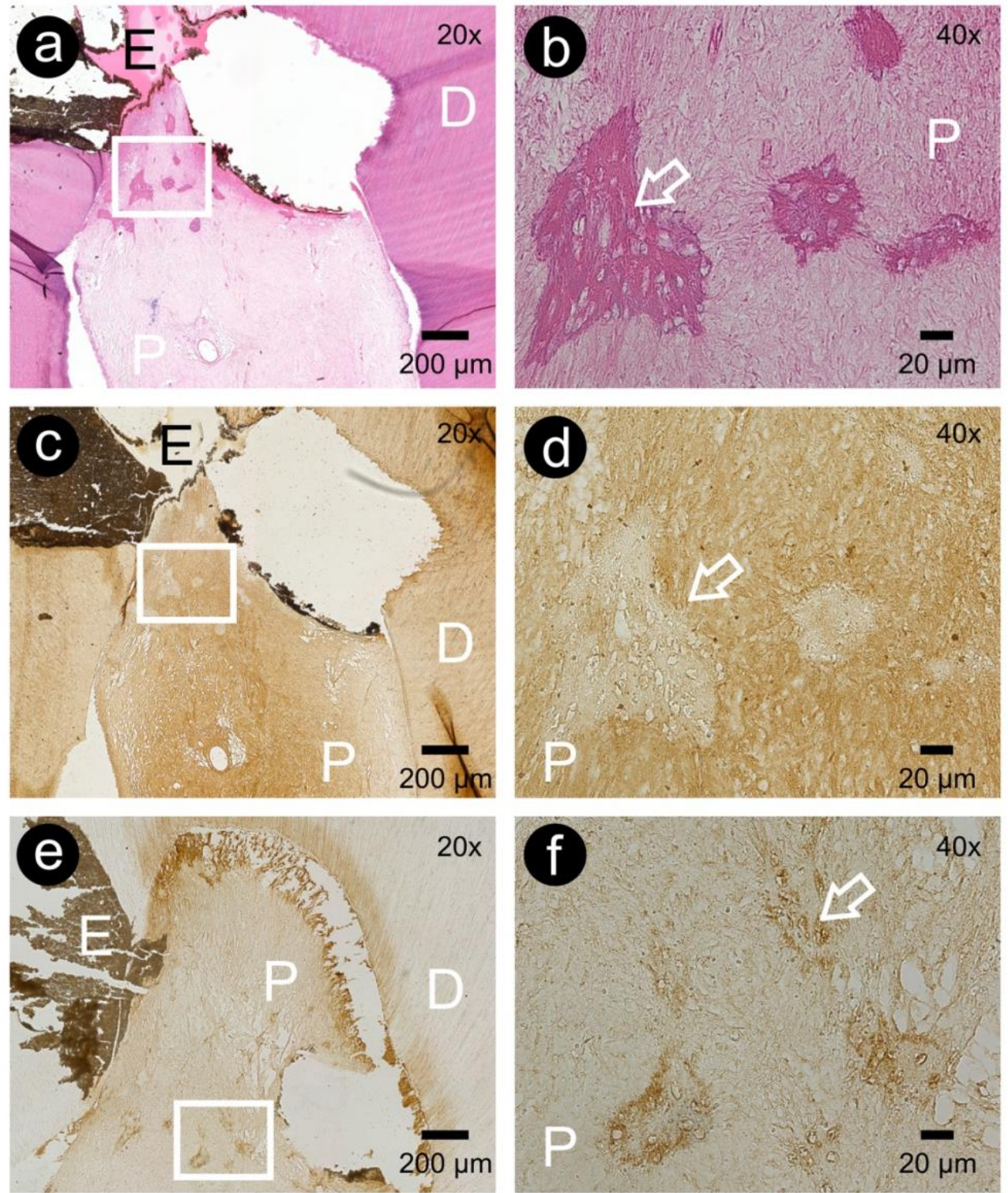
Fig. 8
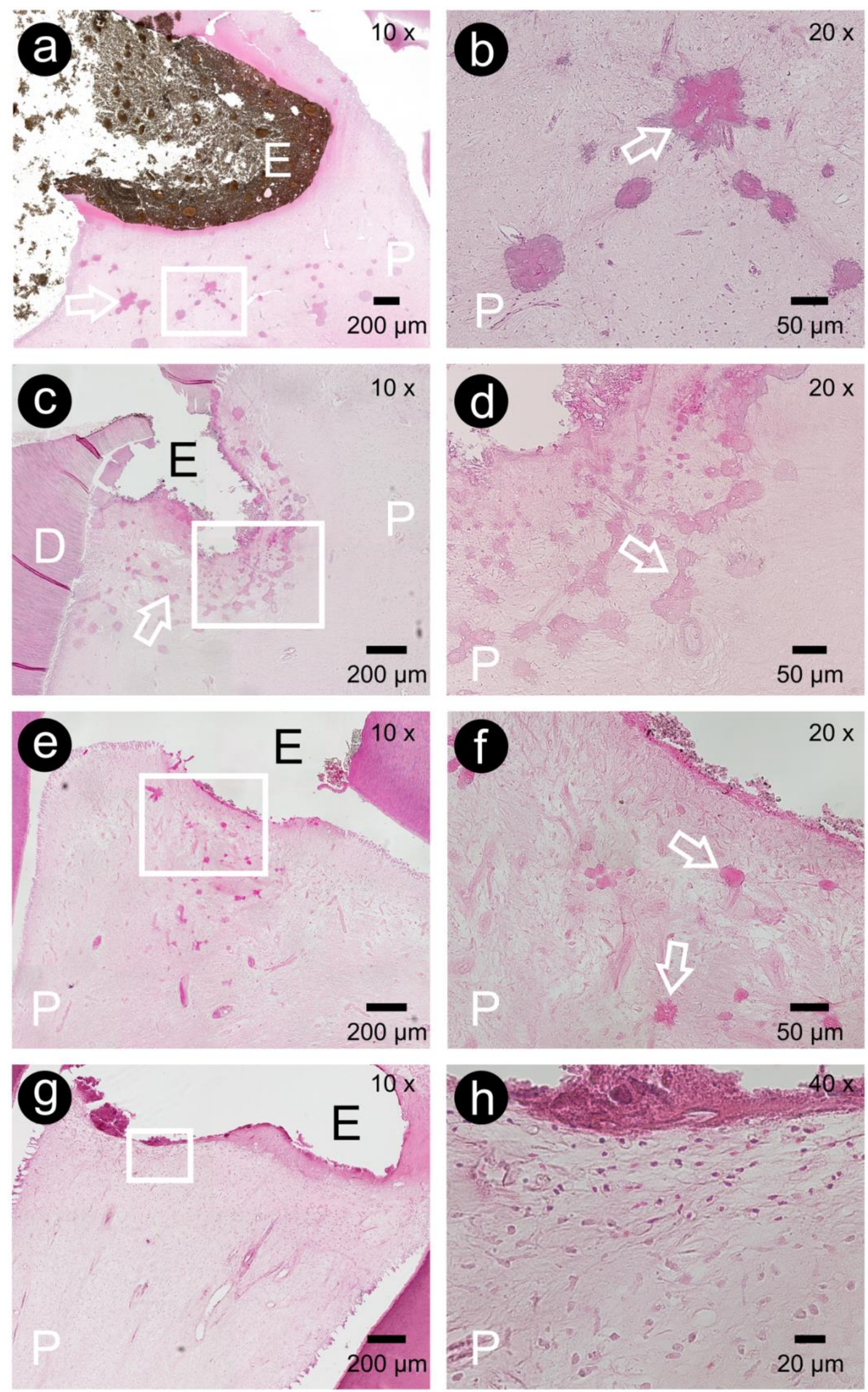

Page $\mathbf{2 9}$ of $\mathbf{2 9}$ 